\title{
A characterization of the invertible measures
}

\author{
by
}

A. ÜLGER (Istanbul)

\begin{abstract}
Let $G$ be a locally compact abelian group and $M(G)$ its measure algebra. Two measures $\mu$ and $\lambda$ are said to be equivalent if there exists an invertible measure $\varpi$ such that $\varpi * \mu=\lambda$. The main result of this note is the following: A measure $\mu$ is invertible iff $|\widehat{\mu}| \geq \varepsilon$ on $\widehat{G}$ for some $\varepsilon>0$ and $\mu$ is equivalent to a measure $\lambda$ of the form $\lambda=a+\theta$, where $a \in L^{1}(G)$ and $\theta \in M(G)$ is an idempotent measure.
\end{abstract}

Introduction. Let $G$ be a locally compact abelian group equipped with its Haar measure, $L^{1}(G)$ its group algebra and $M(G)$ its measure algebra, the multiplication being the convolution. This note concerns the question when a given measure $\mu \in M(G)$ is invertible. As is well-known, an element of a commutative unital Banach algebra is invertible iff the Gelfand transform of that element does not vanish on the Gelfand spectrum of the algebra. Suppose now that $G$ is not discrete. Then the Gelfand spectrum of the algebra $M(G)$ is of the form $\Delta(M(G))=\widehat{G} \cup H$, where $\widehat{G}$ is the dual group of $G$ and $H$ is the hull of $L^{1}(G)$ considered as an ideal in $M(G)$. A classical phenomenon known as the "Wiener-Pitt phenomenon" $[\mathrm{Ru} ; 5.3 .4]$ says that the closure of the set $\widehat{G}$ is not dense in the Gelfand spectrum of $M(G)$. In other words, for a measure $\mu \in M(G)$ to be invertible, the condition " $|\widehat{\mu}| \geq \varepsilon$ on $\widehat{G}$ for some $\varepsilon>0$ " is necessary but far from being sufficient. On the other hand, a characterization of the invertible elements of the algebra $M(G)$ involving a part of its Gelfand spectrum larger than the closure of $\widehat{G}$ in $\Delta(M(G))$ is not considered to be a good characterization. Thus we are faced with a dilemma: How to characterize the invertible elements of the algebra $M(G)$ without involving the unattainable part, $H \backslash \overline{\widehat{G}}$, of its Gelfand spectrum? As far as we know, no such characterization has appeared in the literature. We propose here such a characterization. The main ingredient of the proof of the theorem we have stated in the abstract is a deep result due essentially to B. Host

2000 Mathematics Subject Classification: Primary 43A10, 43A22, 47B48, 46J10.

Key words and phrases: measure algebra, invertible measures, Fourier algebra.

This work of the author is supported in part by the Turkish Academy of Sciences. 
and F. Parreau [Ho-Pa], known as the Glicksberg-Host-Parreau theorem, which states that for a measure $\mu \in M(G)$, the ideal $\mu * L^{1}(G)$ is closed in $L^{1}(G)$ iff $\mu$ is equivalent to an idempotent measure. In Section 2 we present a similar characterization of the invertible elements of the Fourier-Stieltjes algebra $B(G)$ of a locally compact amenable group $G$. For the proof of this result our main ingredient is the analogue of the Glicksberg-Host-Parreau theorem proved for the Fourier algebra $A(G)$ of an amenable locally compact group $G$ in $[\ddot{\mathrm{U}}]$. In the third section these results are extended to some abstract Banach algebras. Our notation and terminology are quite standard and follow the book of $\mathrm{W}$. Rudin [Ru]. Some other notation and terminology will be introduced as and where needed.

1. Invertible measures. Let $G$ be a locally compact abelian group equipped with its Haar measure, $L^{1}(G)$ its group algebra and $M(G)$ its measure algebra. We shall say that two measures $\mu$ and $\lambda$ are equivalent if there exists an invertible measure $\varpi \in M(G)$ such that $\varpi * \mu=\lambda$. This is indeed an equivalence relation on the algebra $M(G)$.

We start by recalling the Glicksberg-Host-Parreau theorem.

Theorem 1.1. Let $\mu \in M(G)$. The ideal $\mu * L^{1}(G)$ is closed in $L^{1}(G)$ iff $\mu$ is equivalent to an idempotent measure.

We shall use this theorem in the following form.

Corollary 1.2. For a noninvertible measure $\mu \in M(G)$, the ideal $\mu *$ $L^{1}(G)$ is closed in $L^{1}(G)$ iff zero is an isolated point of the spectrum $\sigma(\mu)$ of $\mu$.

Proof. If the ideal $\mu * L^{1}(G)$ is closed in $L^{1}(G)$ then, by the preceding theorem, $\mu$ is equivalent to an idempotent measure so that zero is an isolated point of $\sigma(\mu)$. Conversely, if zero is an isolated point of $\sigma(\mu)$ then, by [Lau$\mathrm{Mb}$; Theorem 10] or [Ko; Proposition A], the ideal $\mu * L^{1}(G)$ is closed in $L^{1}(G)$.

Before stating the first main result of the paper, let us recall once more (since we shall use it below) that the Gelfand spectrum $\Delta(M(G))$ of the Banach algebra $M(G)$ is of the form $\Delta(M(G))=\widehat{G} \cup H$, where $H$ is the hull of the ideal $L^{1}(G)$ in the algebra $M(G)$. We identify (via the RadonNikodym theorem) the elements of $L^{1}(G)$ with the measures in $M(G)$ that are absolutely continuous with respect to the Haar measure of $G$.

TheOREM 1.3. A measure $\mu \in M(G)$ is invertible iff $|\widehat{\mu}| \geq \varepsilon$ on $\widehat{G}$ for some $\varepsilon>0$ and $\mu$ is equivalent to a measure $\lambda$ of the form $\lambda=a+\theta$, where $a \in L^{1}(G)$ and $\theta \in M(G)$ is an idempotent measure. 
Proof. Suppose first that $\mu$ is invertible. Then of course $|\widehat{\mu}| \geq \varepsilon$ on $\widehat{G}$ for some $\varepsilon>0$. Also $\mu$, being invertible, is equivalent to the identity element $\delta_{e}$ (the Dirac measure at the unit element $e$ of $G$ ) of the algebra $M(G)$, which is an idempotent. So $\mu$ is equivalent to the measure $\lambda=0+\delta_{e}$, where 0 is the zero element of $L^{1}(G)$.

Conversely, suppose that $|\widehat{\mu}| \geq \varepsilon$ on $\widehat{G}$ for some $\varepsilon>0$ and that $\mu$ is equivalent to $\lambda=a+\theta$, where $a \in L^{1}(G)$ and $\theta$ is an idempotent measure. We want to prove that $0 \notin \sigma(\mu)$. Towards a contradiction, assume that $0 \in \sigma(\mu)$. There are two possibilities: either zero is an isolated point of $\sigma(\mu)$ or an accumulation point of it. If zero is an isolated point of $\sigma(\mu)$ then, by the preceding corollary, the ideal $\mu * L^{1}(G)$ is closed in $L^{1}(G)$. So, by the Glicksberg-Host-Parreau theorem, $\mu=\nu * \varrho$ with $\nu$ idempotent and $\varrho$ invertible. Since $|\widehat{\mu}| \geq \varepsilon$ on $\widehat{G}$, we must have $|\widehat{\nu}| \geq \delta$ on $\widehat{G}$ for some $\delta>0$. Since $\nu$ is an idempotent and $|\widehat{\nu}| \geq \delta$ on $\widehat{G}$, the multiplier $T: L^{1}(G) \rightarrow$ $L^{1}(G)$, defined by $T(a)=\nu * a$, is bijective. Hence $T$ is invertible. This is equivalent to saying that $\nu$ is invertible. Then $\mu=\nu * \varrho$ is invertible, which contradicts the assumption that $0 \in \sigma(\mu)$.

Now assume that zero is an accumulation point of $\sigma(\mu)$. Then, since $\sigma(\mu)=\widehat{\mu}(\Delta(M(G)))$, where $\widehat{\mu}$ is the Gelfand transform of $\mu$, there must exist a sequence $\left(\gamma_{n}\right)_{n \in \mathbb{N}}$ in $\Delta(M(G))$ such that, for each integer $n \in \mathbb{N}$, $\widehat{\mu}\left(\gamma_{n}\right) \neq 0$ and $\widehat{\mu}\left(\gamma_{n}\right) \rightarrow 0$ as $n \rightarrow \infty$. Since $|\widehat{\mu}| \geq \varepsilon$ on $\widehat{G}$, all but finitely many $\gamma_{n}$ 's must be in $H$. As $\mu=a+\theta$, we must have $\widehat{\mu}\left(\gamma_{n}\right)=\widehat{\theta}\left(\gamma_{n}\right) \neq 0$ for all $n \in \mathbb{N}$. As $\theta$ is an idempotent, $\widehat{\theta}\left(\gamma_{n}\right)=1$ whenever $\widehat{\theta}\left(\gamma_{n}\right) \neq 0$. Thus $\widehat{\theta}\left(\gamma_{n}\right)=1$ for all but finitely many $n \in \mathbb{N}$ and $\widehat{\theta}\left(\gamma_{n}\right)=\widehat{\mu}\left(\gamma_{n}\right) \rightarrow 0$ as $n \rightarrow \infty$, which is not possible.

It follows that $0 \notin \sigma(\mu)$, and so $\mu$ is invertible.

Thus, up to equivalence, a measure $\mu \in M(G)$ satisfying the condition " $\delta(\mu)=\inf _{\gamma \in \widehat{G}}|\widehat{\mu}(\gamma)|>0$ " is invertible iff it is a perturbation of an idempotent measure by an element of $L^{1}(G)$. We recall that, as proved in [Ha], if $G$ is not compact, then every measure $\mu \in M(G)$ can be decomposed as $\mu=a+\lambda$, where $a \in L^{1}(G)$ and $\lambda$ has natural spectrum, i.e. $\sigma(\lambda)=\overline{\widehat{\lambda}(\widehat{G})}$. So if $\lambda$ is equivalent to an idempotent measure then $\mu$ is equivalent to a measure of the form $b+\theta$ with $b \in L^{1}(G)$ and $\theta \in M(G)$ idempotent. We also recall that the idempotent elements of $M(G)$ are known in the sense that for $\theta \in M(G)$ to be an idempotent measure it is necessary and sufficient that the Fourier-Stieltjes transform $\hat{\theta}$ of $\theta$ be the characteristic function of a set $E$ belonging to the coset ring $\Re(\widehat{G})$ of $\widehat{G}$ generated by the cosets of the open subgroups of $\widehat{G}[\mathrm{Co}]$. In particular, if $\widehat{G}$ is connected then a measure $\mu \in M(G)$ is invertible iff $\delta(\mu)>0$ and $\mu$ is equivalent to a measure of the form $\lambda=a+\delta_{e}$, where $a \in L^{1}(G)$. 
Without using the above equivalence relation, Theorem 1.3 above can be restated as follows.

Theorem 1.4. A measure $\mu \in M(G)$ is invertible iff $\delta(\mu)=\inf _{\gamma \in \widehat{G}}|\widehat{\mu}(\gamma)|$ $>0$ and $\mu=a+\lambda$, where $a \in L^{1}(G)$ and $\lambda \in M(G)$ is such that the ideal $\lambda * L^{1}(G)$ is closed in $L^{1}(G)$.

2. Invertible elements of the Fourier-Stieltjes algebras $B(G)$. Let $G$ be a locally compact group. In [Ey] P. Eymard associated to $G$ two Banach algebras, $A(G)$ and $B(G)$, known as the Fourier algebra and FourierStieltjes algebra of $G$, respectively. These are commutative semisimple Banach function algebras with pointwise multiplication. The algebra $B(G)$ is always unital whereas $A(G)$ is unital only if $G$ is compact, in which case $A(G)=B(G)$. As sets, $A(G) \subseteq C_{0}(G)$ and $B(G) \subseteq C_{\mathrm{b}}(G)$, the space of continuous bounded functions $u: G \rightarrow \mathbb{C}$. For the properties of these algebras we refer the reader to the seminal paper of P. Eymard [Ey] and to the book [Pi] of J.-P. Pier. Let us just mention that: 1) The algebra $A(G)$ has a BAI (= bounded approximate identity) iff $G$ is amenable. 2) $A(G)$ is a closed ideal of $B(G)$. 3) The Gelfand spectrum of $A(G)$ is $G$ and each $\gamma \in G$ acts as a Dirac measure on the elements of $A(G)$. 4) If $G$ is amenable, then $B(G)$ is the multiplier algebra of $A(G) .5)$ If $G$ is commutative, then $A(G)=L^{1}(\widehat{G})$ (via Fourier transform) and $B(G)=M(\widehat{G})$ (via Fourier-Stieltjes transform).

As above, we shall say that two elements $u$ and $v$ of the algebra $B(G)$ are equivalent if, for some invertible element $p$ of $B(G)$, we have $u=p . v$.

Throughout this section $G$ will be a locally compact amenable group. We shall need the following result proved in [ت̈; Theorem 2.7 or Corollary 3.8].

Theorem 2.1. Let $u \in B(G)$ be a given element. Then the ideal $u . A(G)$ $=\{u . a: a \in A(G)\}$ is closed in $A(G)$ iff $u$ is equivalent to an idempotent element of $B(G)$.

At this point we recall that an element $p$ of $B(G)$ is equivalent to an idempotent element of $B(G)$ iff, as in the commutative case, the set $E=$ $\{\gamma \in G: p(\gamma) \neq 0\}$ belongs to the coset $\operatorname{ring} \Re(G)$ of the group $G$ generated by the cosets of the open subgroups of $G$ [Ho].

The proof of Corollary 1.2 above shows that the following analogue of that corollary also holds for the algebra $A(G)$ since Theorem 10 of [Lau$\mathrm{Mb}$ ] that we have used to prove Corollary 1.2 is valid for any semisimple Banach algebra.

Corollary 2.2. For a noninvertible element $u \in B(G)$, the ideal $u . A(G)$ is closed in $A(G)$ iff zero is an isolated point of the spectrum $\sigma(u)$ of $u$. 
The proof of the next theorem, which is the second main result of this note, is just an adaptation of the proof of Theorem 1.3 above. For this reason we shall omit it.

Theorem 2.3. An element $u$ of $B(G)$ is invertible iff $|u| \geq \varepsilon$ on $G$ for some $\varepsilon>0$ and $u$ is equivalent to an element $v$ of the form $v=a+\theta$, where $a \in A(G)$ and $\theta$ is an idempotent element of $B(G)$.

We could deduce Theorem 1.3 from the preceding theorem since the proof of Theorem 2.1 above is completely independent of the proof of the Glicksberg-Host-Parreau theorem. We preferred to present Theorem 1.3 and Theorem 2.3 separately essentially for two reasons. First, the GlicksbergHost-Parreau theorem is a famous theorem of historical importance; we could not bypass it. Secondly, the measure algebra $M(G)$ is an object better known than the Fourier-Stieltjes algebra $B(G)$ of an amenable group $G$. This is also the reason for the choice of the paper's title.

3. The case of some abstract Banach algebras. We proceed, contrary to the general tendency in mathematics, from the concrete to the abstract; we think that this approach is more pedagogical. Moreover, the abstract result presented below does not cover the main results of the preceding sections. The essential reason is that the analogue of the GlicksbergHost-Parreau theorem is not valid for general commutative semisimple Banach algebras. For instance, if $A$ is the classical disk algebra and $u(z)=z$ then the ideal u.A $=\{a \in A: a(0)=0\}$ is closed in $A$ but $u$ is not equivalent to an idempotent element of this algebra since $u$ is not invertible and the only idempotent element of $A$ is its identity. The class of Banach algebras we are interested in in this section is the class of commutative semisimple Tauberian Banach algebras $A$ with a BAI and with discrete spectrum. The term "Tauberian" means that the set $\{a \in A: \operatorname{supp} \widehat{a}$ is compact $\}$ is norm dense in $A$. For instance, any quotient algebra of $L^{1}(G)$ ( $G$ compact abelian) and the algebras $c_{0}, c_{0} \widehat{\otimes} c_{0}, c_{0} \widehat{\otimes} A(G)$ ( $G$ discrete amenable) belong to this class. The essential fact about Banach algebras belonging to this class is that the analogue of the Glicksberg-Host-Parreau theorem holds for them.

In this section, $A$ will denote a commutative semisimple Tauberian Banach algebra with a BAI and discrete spectrum. We recall that a linear operator $T: A \rightarrow A$ is said to be a multiplier if $T(a b)=a T(b)$ for all $a$ and $b$ in $A$. The space $M(A)$ of multipliers of $A$, under the operator norm and the composition of operators, is a commutative semisimple unital Banach algebra [La]. As above we shall say that two multipliers $T$ and $S$ in $M(A)$ are equivalent iff $R \circ T=S$ for some invertible element $R$ of $M(A)$. 
For $a \in A, L_{a}: A \rightarrow A$ is the multiplier defined by $L_{a}(b)=a b$. The following result is proved in [ $\ddot{\mathrm{U}}$; Theorem 3.9].

Theorem 3.1. A multiplier $T: A \rightarrow A$ has a closed range iff $T$ is equivalent to an idempotent multiplier.

The proofs of the following two results are the same as the proofs of their counterparts in Section 1. For that reason we state them without proof.

Corollary 3.2. The range of a noninvertible multiplier $T: A \rightarrow A$ is closed in A iff zero is an isolated point of $\sigma(T)$.

THEOREM 3.3. A multiplier $T \in M(A)$ is invertible iff $|\widehat{T}| \geq \varepsilon$ on $\Delta(A)$ for some $\varepsilon>0$ and $T$ is equivalent to a multiplier of the form $T=L_{a}+\theta$, where $a \in A$ and $\theta \in M(A)$ is idempotent.

From the results presented above it is clear that, if $A$ is any commutative semisimple Banach algebra with a BAI for which the analogue of the Glicksberg-Host-Parreau theorem holds, then the exact analogue of the preceding theorem holds for the multipliers of this algebra. The characterization of the commutative Banach algebras with a BAI for which the analogue of the Glicksberg-Host-Parreau theorem holds seems to be an open problem. For some examples of Banach algebras for which the analogue of the Glicksberg-Host-Parreau theorem holds we refer the reader to the paper $[\ddot{\mathrm{U}}]$.

\section{References}

[Co] P. Cohen, On a conjecture of Littlewood and idempotent measures, Amer. J. Math. 82 (1960), 191-212.

[Ey] P. Eymard, L'algèbre de Fourier d'un groupe localement compact, Bull. Soc. Math. France 92 (1964), 181-236.

[Ha] O. Hatori, Measures with natural spectra on locally compact abelian groups, Proc. Amer. Math. Soc. 126 (1998), 2351-53.

[Ho] B. Host, Le théorème des idempotents dans $B(G)$, Bull. Soc. Math. France 114 (1986), 215-223.

[Ho-Pa] B. Host et F. Parreau, Sur un problème de I. Glicksberg: Les idéaux fermés de type fini de $M(G)$, Ann. Inst. Fourier (Grenoble) 28 (1978), no. 3, 143-164.

[Ko] J. J. Koliha, Isolated spectral points, Proc. Amer. Math. Soc. 124 (1996), 3417-3424.

[La] R. Larsen, The Theory of Multipliers, Springer, Berlin, 1971.

[Lau-Mb] K. B. Laursen and M. Mbekhta, Closed range multipliers and generalized inverses, Studia Math. 107 (1993), 127-135.

[Pi] J.-P. Pier, Amenable Locally Compact Groups, Wiley-Interscience, 1984.

[Ru] W. Rudin, Fourier Analysis on Groups, Interscience, New York, 1962. 
[ய̈] A. Ülger, A characterization of the closed unital ideals of the Fourier-Stieltjes algebra $B(G)$ of a locally compact amenable group $G$, J. Funct. Anal. 205 (2003), 90-106.

Department of Mathematics

Koc University

Fener Yolu, 34450, Sariyer-Istanbul, Turkey

E-mail: aulger@ku.edu.tr

Received October 7, 2006

Revised version August 7, $200 \%$ 